doi: https://doi.org/10.5377/paradigma.v28i46.12836

\title{
Capital Cultural en Estudiantes Universitarios de Honduras: Una Comparación con el Rendimiento Académico, Nivel Educativo e Ingreso Económico de sus Padres
}

\section{Cultural Capital in Honduran University Students: A Comparison with the Academic Performance, Educational Level and Economic Income of their Parents}

\author{
Dani Oved Ochoa Cervantez ${ }^{1}$ \\ Luis Eduardo Alvarenga Aguilera ${ }^{2}$
}

\begin{abstract}
Resumen
En el contexto de los estudiantes universitarios, este estudio analiza el capital cultural desde las tres formas que sugiere Bourdieu; incorporado, institucionalizado y objetivado, lleva a cabo comparaciones entre éstas y tres variables categorías; el rendimiento académico, nivel educativo e ingreso económico de los padres. En la muestra participaron siete carreras e incluyó a 735 estudiantes de tres universidades hondureñas. Para la medición de las variables capital cultural incorporado e institucionalizado se construyó un instrumento tipo Likert, por la naturaleza del capital cultural objetivado se utilizó una escala nominal. El Alpha de Cronbach se utilizó para medir la confiabilidad, el capital cultural incorporado e institucionalizado presentaron valores de .817 y 0.763 . Las pruebas estadísticas de Kruskal-Wallis mostraron p-valor:0.000< $<: 0.05$, evidenciando diferencias de medianas estadísticamente significativas en los niveles de capital cultural incorporado e institucionalizado a partir del rendimiento académico, de manera similar, se encontró diferencia a partir de grado educativo e ingreso de los padres, entre tanto, el capital objetivado parece no estar asociado con el rendimiento académico ( $\mathrm{p}$-valor:0.000>a:0.05), sin embargo, se encontró estar asociado con el nivel educativo de los padres e ingresos económicos. El estudio concluye que el capital cultural que muestran los estudiantes es un factor importante para el éxito educativo en el contexto universitario.

Palabras clave: Capital Cultural, rendimiento académico, nivel educativo e ingresos económicos de los padres, educación superior, Honduras
\end{abstract}

\footnotetext{
${ }^{1}$ d.ochoa@unacifor.edu.hn. Universidad Nacional de Ciencias Forestales. Siguatepeque, Honduras. https://orcid.org/0000-0002-7707-9461

${ }^{2}$ lalvarenga@upnfm.edu.hn. Universidad Pedagógica Nacional Francisco Morazán. Tegucigalpa, Honduras. https://orcid.org/0000-0002-3065-5951
} 


\begin{abstract}
In the context of university students, this study analyzes cultural capital from the three forms suggested by Bourdieu; incorporated, institutionalized and objectified, it carries out comparisons between these and three variable categories; the academic performance, educational level and income of the parents. Seven majors participated in the sample and included 735 students from three Honduran universities. For the measurement of the variables incorporated and institutionalized cultural capital, a Likert-type instrument was constructed; due to the nature of objectified cultural capital, a nominal scale was used. Cronbach's Alpha was used to measure reliability, the incorporated and institutionalized cultural capital presented values of .817 and 0.763 . The Kruskal-Wallis statistical tests showed p-value: $0.000<\alpha: 0.05$, showing statistically significant differences of medians in the levels of incorporated and institutionalized cultural capital based on academic performance, similarly a difference was found from educational grade and income of the parents, meanwhile, the objective capital seems not to be associated with academic performance ( $p$-value: $0.000>\alpha$ : 0.05), however, it was found to be associated with the educational level of the parents and economic income. The study concludes that the cultural capital shown by students is an important factor for educational success in the university context.

Keywords: Cultural capital, academic performance, educational level and income of parents, higher education, Honduras.
\end{abstract}

\title{
Introducción
}

Las familias proporcionan a sus hijos símbolos culturales de alto estatus que facilitan la selección social, así, las familias más privilegiadas enseñan a sus hijos a cultivar ciertas preferencias, comportamientos y actitudes que las instituciones educativas y las organizaciones en general, valoran y recompensan (Bourdieu, 1987), esto produce desigualdades académicas y económicas entre los hijos de aquellas familias que pueden y las que no pueden proporcionar los recursos necesarios para cultivar estos comportamientos en sus hijos (Wu y Bai, 2015; Carolan y Wasserman, 2015).

Bajo estos planteamientos, el sociólogo Bourdieu (1986), sugiere que las instituciones educativas son espacios donde se observa una reproducción cultural que tiene efectos desde el punto de vista social y económico en los estudiantes, a los que él llama agentes. Así, cuando el capital cultural, en adelante $\mathrm{CC}$, que muestra un agente se ajusta con el que privilegian las instituciones educativas, existe la posibilidad que tengan un mayor éxito educativo (Breinhol y Jæger, 2020). El problema radica en que los capitales que se promueven en los hogares difieren del favorecido y valorado en las instituciones educativas generando una desvinculación entre lo enseñado y su identidad. 
En esta misma línea de pensamiento, es cierto que los planteamientos de Bourdieu sobre el CC se centraron en el contexto del sistema educativo francés, sin embargo, hay evidencia de investigaciones internacionales que han estudiado la variable en los sistemas educativos en diversos países, incluso en el nivel universitario (Mohammadinia y Negahdari, 2017; Košutić, 2017), de allí que, el CC es un fenómeno universal (Dumais, 2015), y sus hallazgos varían según la nación y, a menudo, incluso dentro de los países.

A pesar que las investigaciones realizadas recientemente y su asociación con otras variables, por ejemplo, con el rendimiento académico (Cheng y Kaplowitz, 2016), educación de los padres de los estudiantes (Cheng y Kaplowitz, 2016; Suhonen y Karhunen, 2019 y Yong Tan; Peng y Lyu, 2019), ingresos económicos (Negewo y Murugan, 2018), la revisión de la literatura deja en evidencia que existen escasos estudios donde se haya abordado esta asociación desde las formas que plantea Bourdieu; incorporado, institucionalizado y objetivado, lo anterior constituye un espacio de oportunidad para aportar al conocimiento científico.

El planteamiento anterior deja en evidencia que la propuesta para el abordaje de estos tres estados no ha sido analizada en el contexto universitario hondureño desde una perspectiva cuantitativa inferencial de alcance comparativo, este hecho constituye otra razón para realizar este estudio. Basado en lo anterior, este estudio se cuestiona sobre el siguiente vacío de conocimiento encontrado desde la perspectiva del investigador: ¿Los niveles de CC de los estudiantes, en sus tres estados, son distintos según los niveles de rendimiento académico, el nivel educativo de sus padres e ingresos? El objetivo general de la investigación fue determinar diferencias estadísticas en los niveles de los estados del CC a partir de su comparación con los niveles de rendimiento académico, educación de sus padres e ingresos familiares.

Se plantearon cuatro objetivos específicos, primero, describir los niveles de CC; Incorporado, Institucionalizado y Objetivado que muestran los estudiantes, segundo, determinar la diferencia estadística en el CC entre los niveles de rendimiento académico, tercero, determinar la diferencia estadística en el CC que muestran los estudiantes entre los niveles educativos de sus padres y cuarto, determinar la diferencia estadística en el CC que muestran los estudiantes entre los niveles de ingresos económicos observados.

El articulo está estructurado en diferentes secciones, en primer lugar, la construcción del objeto de estudio; problema de investigación, pregunta y objetivos, seguidamente se plantea una revisión teórica relevante de la temática, procurando la realización de comparaciones entre los diferentes 
postulados y elaborando críticas fundamentadas para identificar los límites de la teoría, luego se explica el abordaje metodológico del estudio que orientó el desarrollo de la investigación, en cuarto lugar, se muestran los resultados que ofrecen una respuesta a los objetivos e hipótesis planteadas, finalmente se hace una discusión de los resultados, se elaboran las conclusiones y se sugieren recomendaciones sustentadas en los hallazgos.

\section{Discusión Teórica}

\section{Teoría de la Reproducción Social}

La teoría de la reproducción social de Bourdieu (1997) expone que el CC es utilizado por grupos favorecidos para promover la reproducción social, en consecuencia, los grupos socioeconómicamente desfavorecidos no lo poseen y, si lo tienen, no son igualmente capaces de beneficiarse de éste. Desde esta teoría, las familias que tienen un alto nivel socioeconómico poseen un mayor CC, por tanto, los padres transmiten este capital a sus hijos a quienes les permite tener una posición dominante y favorecer la preproducción social (Negewo y Murugan, 2018).

Bourdieu (1977) se centra en los aspectos culturales de la desigualdad social, así como en los mecanismos sociales que crean y perpetúan la reproducción social y permiten el mantenimiento de una sociedad clasificada. Esta reproducción social genera una legitimización de las desigualdades sociales mediante la reproducción de las clases dominantes e identifica mecanismos de reproducción social que se perpetúan, por ejemplo, la transferencia de la desigualdad económica y sostiene que la reproducción de la cultura de las clases dominantes promueve las desigualdades sociales.

\section{Habitus, Campos, Espacio Social, Juego}

Uno de los conceptos abordados por Bourdieu (1977), es el habitus, que lo define como: “un sistema de disposiciones duraderas y transponibles que, integrando experiencias pasadas, funciona en cada momento como una matriz de percepciones, apreciaciones y acciones" (p. 82) parafraseando al autor, se entiende el habitus como las disposiciones internas que tiene un individuo que generan prácticas dentro del campo. Si bien el volumen de capital de un individuo puede determinar su posición en el campo, su habitus determina su disposición hacia el campo (Bourdieu y Wacquant, 1992).

El habitus hace que un grupo social (clase) se convierta justamente en ello, es decir, el habitus produce decisiones y acciones individuales en cada miembro de un grupo social o clase y cuando se trata de objetos y situaciones específicas, se parezcan entre sí. El habitus es una "orquestación sin director que da regularidad, unidad y sistematización a las prácticas de un grupo o clase, y esto incluso en ausencia de cualquier organización espontánea o impuesta externamente de proyectos 
individuales" (Bourdieu, 1977, p. 80). De allí que, "las prácticas de los miembros de un mismo grupo o clase están más y mejor armonizadas de lo que los agentes saben o desean” (Bourdieu 1977, p. 81).

Por otro lado, argumenta sobre el concepto de espacio social, sugiere que es la idea de diferencia, separación, posiciones coexistentes aglutinadas, pero también distintas. Bourdieu retrata la sociedad moderna a partir de la imagen de un espacio social en el que los agentes se ubican en distintas posiciones relativas, según la distribución de propiedades entre ellos. Estas propiedades se forjan como recursos de poder y se refieren a diferentes formas de capital que pueden ser apropiadas por los agentes.

Finalmente, sobre los campos, el autor sostiene que son mundos sociales que corresponden a leyes sociales más o menos específicas. Todos los campos son campos de fuerza y disputa, y la posición que adopte cada agente en la estructura de distribución del capital específico de cada campo conducirá sus posibilidades de acción.

\section{Habitus en lo Educativo}

Desde el contexto educativo, el habitus explica como las diversas posiciones de clase entre las familias generan varios sistemas de pensamiento y razonamiento, es decir, una forma concreta de pensar y esto constituye el habitus. El habitus que adquieren las familias es relevante debido a que constituye el fundamento para aceptar y comprender los códigos en el aula. Es decir, el habitus predispone a los estudiantes hacia el sistema educativo para mostrar una determinada actitud, invertir tiempo, esfuerzo y recursos económicos para mantener su capital heredado y el estatus familiar.

Es el mecanismo que media entre estructura y agencia, los estudiantes internalizan el entorno en el que se encuentran y emiten juicios sobre lo que es posible y realista para ellos, entonces, los antecedentes familiares de un estudiante desempeñen un papel integral en la configuración del habitus, aquellos que están familiarizadas con la universidad o tienen un historial de trabajo en campos relacionados con la ciencia pueden tener más probabilidades de tener disposiciones internalizadas y ven la educación como algo importante.

En este orden de ideas, el habitus de un estudiante está influenciado por su contexto familiar (Dimaggio, 1982). Aunado a ello, algunos teóricos señalan el concepto de "habitus familiar" como una herramienta para comprender cómo los jóvenes internalizan los recursos, valores y opciones de estilo de vida de la familia (Archer et al., 2012). Si bien la familia proporciona un contexto que propicia el habitus, éste también está conformado por grupos culturales más amplios con las que el individuo se identifica, y sus experiencias en otros contextos, como la escuela. Bourdieu (1984, p. 101) afirma que, si los individuos están expuestos a “condiciones de existencia homogéneas”, 
es decir, experiencias de vida similares, tendrán habitus similares. En este sentido, el habitus puede adquirir una cualidad colectiva donde los miembros de un mismo grupo socializan de manera similar, predisponiéndolos a tener disposiciones similares.

\section{Teoría del Capital de Bourdieu}

Bourdieu (1997), reconoce diferentes formas de capital (ver Figura 1), sugiere un capital económico que es la posesión de activos materiales y financieros, además constituye el control de los recursos económicos en una sociedad determinada, por ejemplo, la propiedad privada de bienes muebles e inmuebles; el dominio de las ofertas de bienes; y servicios y dirección de empresas. El capital social consiste en recursos acumulados en virtud de pertenecer a un grupo, es "la suma de los recursos reales o potenciales que están vinculados a la posesión de una red duradera o relaciones más o menos institucionalizadas de conocimiento o reconocimiento mutuos” (Bourdieu, 1997, p. 51), es este sentido, constituye un conjunto de relaciones producidas en el transcurso del tiempo que genera reconocimiento, autoridad, prestigio, influencia, etc.

El capital simbólico, designa los efectos de cualquier forma de capital cuando la gente no los percibe como tales, sugiere la capacidad de imponer una visión concreta del mundo, siempre anclada en una división social (ellos y nosotros), a través de un tipo de lenguaje legítimo con la intención de hacerse de la verdad y, además, se utiliza para legitimar la propiedad de otras formas de capital y la violencia simbólica contra las clases sociales más bajas. Finalmente, el capital cultural comprende recursos y competencias disponibles que son movilizables cuando se trata de la cultura dominante, constituyen bienes, habilidades y títulos simbólicos escasos, hace referencia a los recursos no financieros de un individuo, como los objetos que posee (por ejemplo, libros, ropa, muebles) o las características que encarna (por ejemplo, acento, postura).

\section{Figura 1}

Tipos de capital según Bourdieu

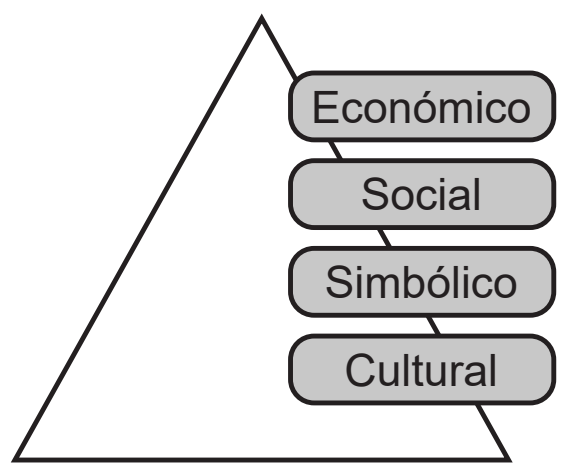

Nota. Fuente: (Bourdieu, 1997). 
El autor enfatiza que los capitales son recursos que generan luchas simbólicas entre los agentes en diferentes campos y es posible que se transmitan o conviertan, bajo estos planteamientos, los distintos capitales son recolectados y transmitidos como herencia por miembros de diferentes clases sociales a la próxima generación y pueden usarse para producir o reproducir patrones de desigualdad y consumo.

Por la naturaleza de esta investigación se profundiza en los planteamientos teóricos del CC, Dumais (2015) sugiere que éste es el capital que tiene más implicaciones en los sistemas educativos y constituye un poder simbólico que mediado por la educación se puede transformar en capital económico (Bourdieu, 1997).

\section{Capital Cultural}

El CC se refiere a recursos no financieros que un individuo tiene a su disposición (Bourdieu, 1984, 1986, 1997), es el instrumento que permite agenciarse de bienes simbólicos que las sociedades definen como deseados y poseídos, desde esta visión, se entiende, como la apropiación de riquezas simbólicas que la sociedad designa como valiosa y que, por tanto, se deben buscar y poseer, para el autor, existen tres estados o formas de percibirse; estado incorporado, institucionalizada y objetivado, en la sección siguiente se conceptualiza cada uno de ellos.

\section{Capital Cultural Incorporado}

Es el CC más complicado a adquirir por los dominados, porque existe una brecha entre aprender sobre música o artes y expresar preferencia y disfrutarlos (Bourdieu, 1987). Así, este capital se adquiere producto de socializar con patrones culturales que principalmente están en el núcleo de la familia.

Como señala Bourdieu (1987):

...quien lo posee ha pagado con su persona, con lo que tiene de más personal: su tiempo.

Este capital personal no puede ser transmitido instantáneamente [...], por el don o por la transmisión hereditaria, la compra o el intercambio (p.12).

El mismo autor argumenta que este capital está encarnado en las personas, son afinidades culturales y la disposición de apreciar y comprender el capital objetivado, sugiere diferentes niveles de interés por la música o lecciones de arte o danza, disposición estética y constituyen recursos que se adquieren conscientemente mediante la inversión personal de tiempo y esfuerzo y se hereda pasivamente por la socialización familiar en una cultura dominante a través del habitus. 


\section{Capital Cultural Institucionalizado}

Se refiere al sistema de credenciales educativas, acreditación de formación y avance intelectual, sugiere la posesión del agente de títulos profesionales, calificaciones académicas, diplomas y reconocimiento adquiridos y socialmente reconocibles (Bourdieu, 1997), es decir, acredita la posesión de conocimiento intelectual que certifica estar apto para su desempeño en la sociedad. Este tipo de capital es importante en el mercado laboral porque permite hacer comparaciones, por ejemplo, entre los solicitantes de empleo (Klimczuk, 2015).

\section{Capital Cultural Objetivado}

Constituye recursos físicos que la familia posee en el hogar, entre ellos, material de lectura, espacios físicos que facilitan el aprendizaje, elementos culturales, por ejemplo, literatura, esculturas, música y obras de arte, es una forma material que se relaciona con poseer bienes culturales, refiere a lo que se considera arte elevado y tiende a encontrarse en museos, salas de conciertos y las casas de las clases altas y que requieren habilidades culturales especiales para su uso y apreciación y además, pueden convertirse en capital económico comprándolos y vendiéndolos, o que cuando se usan y consumen, las personas pueden encarnarlos (Bourdieu, 1997).

\section{Transmisión del Capital Cultural desde la Familia}

Desde la visión de Bourdieu (1987), el CC es heredado por las familias del agente, permitiendo formar un conjunto de disposiciones, de gustos y mecanismos de socialización; lo cual, consolida en los agentes un esquema de saberes y "saber hacer" que le puede traer réditos en su paso por las instituciones educativas (Castillo, 2018). Bajo este planteamiento, es evidente que se produce una desigualdad en la distribución, debido, por un lado, a que no todas las familias poseen los mismos niveles capital para transmitirlos o heredarlos al agente y por otro, incluso entre esos niveles, existen diferencias en su legitimización. Esta herencia es vista como una estrategia que consolida la reproducción y ocurre de forma invisible y da como resultado que aquellos agentes que recibieron o heredaron e iniciaron la acumulación de CC en los primeros años de su vida, se conviertan en potenciales familias con igual o superior acumulación de CC.

Uno de los aspectos que se aborda para evaluar la trasmisión de CC, es la educación de los padres de familia, por ejemplo, ésta ayuda a operacionalizar la idea de que el alumno incorpore a través del habitus, los frutos del éxito académico de la familia.

En el campo de la educación, lo planteado anteriormente, genera que las familias con alto $\mathrm{CC}$ realicen inversiones a largo plazo buscando una habilidad que le permita al agente heredar 
y agenciarse de capitales. Así mismo, combinan el CC con el económico con la finalidad de llevar un proceso de “conversión y reconversión” (Castillo, 2019, p. 38), que busca movilizar o mantener al agente en un campo deseado. Los recursos disponibles para los estudiantes a través de su familia son importantes, además de ofrecer formas objetivadas de $\mathrm{CC}$, ofrecen exposición a formas de pensamiento y comprensión que históricamente han sido valoradas por las instituciones educativas.

\section{Capital Cultural: una Visión desde la Universidad}

Para Bourdieu (1997), el sistema educativo presupone la posesión de CC, que solo posee una minoría de estudiantes, provocando ineficiencia en la transmisión pedagógica debido a que los estudiantes simplemente no comprenden lo que sus maestros están tratando de transmitir. Para Bourdieu, esto es particularmente evidente en las universidades, donde los estudiantes, temerosos de revelar el alcance de su ignorancia "minimizan los riesgos arrojando una cortina de humo de vaguedad sobre la posibilidad de verdad o error" (Bourdieu y Passeron, 1977, p. 114).

Al ofrecer explícitamente a todos lo que implícitamente exige de todos, el sistema educativo exige a todos por igual que tengan lo que no les da, olvidando que la posesión de CC varía con la clase social y generando dificultad para alumnos de clases bajas tener éxito en el sistema educativo. A pesar que los alumnos de clases bajas se encuentran en una desventaja en la competencia por las credenciales educativas, los resultados de esta competencia se consideran meritocráticos y, por lo tanto, legítimos. Además, las desigualdades sociales están legitimadas por las credenciales educativas de quienes ocupan posiciones dominantes, por tanto, el sistema educativo tiene un papel clave en el mantenimiento del status quo.

En palabras del autor, la educación:

Es de hecho uno de los medios más efectivos de perpetuar el patrón social existente, ya que proporciona una aparente justificación de las desigualdades sociales y reconoce el patrimonio cultural, es decir, un regalo social tratado como natural (Bourdieu, 1974, p. 32)

La opinión de Bourdieu es que el CC se inculca en el hogar de clase alta y permite a éste obtener credenciales educativas más altas que el estudiante de clase baja, permitiendo que los individuos de clase alta mantengan sus posiciones de clase y así legitimar las posiciones dominantes que normalmente ostentan. 


\section{Capital Cultural y Rendimiento Académico}

De acuerdo con la teoría del CC (Bourdieu, 1986), las variaciones en el rendimiento académico de los estudiantes pueden reflejar las variaciones en el rendimiento académico de los estudiantes pueden reflejar, además de habilidades, los niveles de competencias culturales de alto o bajo estatus que posee el estudiante. Este CC es sancionado arbitrariamente por las instituciones educativas, reforzado por directores y transmitido por los padres con un nivel socioeconómico más alto para perpetuar su ventaja social.

Estos argumentos sugieren que el rendimiento educativo está determinado por el CC previamente invertido en el núcleo familiar, desde esta visión, los estudiantes que provienen de estratos culturalmente favorecidos encuentran facilidad para hacer frente a sus labores educativas, porque son vistas como una continuidad de la educación recibida en la familia, entre tanto, aquellos estudiante cuyas familiar son desfavorecidas culturalmente, las actividades académicas en las instituciones educativas representan algo extraño, distante y amenazador.

Así, el CC se relaciona con el rendimiento académico debido a que la familia lo transmite, bien de forma pasiva, a través de la exposición al capital cultural materializado y encarnado de los padres en el hogar, o activamente por medio de la inversión de los padres en la transmisión de su CC a los niños (Lareau, 2011), Así, se hereda de los padres y se convierte en algo integral de su disposición, lo que Bourdieu llama habitus.

El CC es un recurso particularmente valioso dentro del campo de la educación (Bourdieu, 1977, 1984; Bourdieu y Passeron, 1990). Los sistemas de educación están sesgados hacia la valorización del CC y atribuyen cualidades verdaderas como la brillantez académica a quienes lo poseen. Desde este escenario, quienes lo poseen siguen el ritmo de los sistemas educativos y se muestran académicamente más brillantes ante los actores de la escuela (profesores, compañeros), por tanto, obtienen retornos visibles de la posesión de este capital, que se traduce en un mejor rendimiento académico al parecer más talentosos que los demás.

\section{Teoría de las Capacidades de Amartya Sen}

Uno de los aportes de Sen (2000), es el relacionado con las capacidades, afirma que el Estado debe orientar su esfuerzo en generar una igualdad en las capacidades y no en las oportunidades, para el autor, esta última no garantiza la equidad, sino que promueve la inequidad, cuando se considera a todos por igual produce un trato desigual a los más desfavorables, por tanto, no basta con la igualdad formal(Sen, 2000). 
Desde la mirada de justicia con equidad del autor, el Estado debe procurar que cada persona busque el desarrollo de sus capacidades para alcanzar lo que él desea en libertad, desde este enfoque, para aquellos grupos desfavorecidos un aumento en los medios y recursos aumenta la libertad de éstos para desarrollarse, en cambio, igual en medios y recursos no implica una igualdad en libertad, o bien, que las personas se desarrollen de forma similar (Sen, 2000).

A partir de estos planteamientos, igualdad no es sinónimo de equidad, la primera sugiere un tratamiento igual para todos en función a las oportunidades y meritocracia, entre tanto, la segunda reconoce que cada individuo posee condiciones particulares, por lo tanto, merece un tratamiento diferente.

\section{Métodos y Materiales}

Es un estudio de corte cuantitativo, recolecta los datos para medir relaciones de tipo numérico entre las variables, hace uso de la estadística descriptiva y de la inferencial para la verificación de hipótesis. La investigación tiene un alcance descriptivo, correlacional-causal debido a que busca encontrar relaciones de casualidad entre las variables, por otro lado, mantiene un diseño no experimental transaccional.

Participaron en el estudio tres universidades, 735 estudiantes de siete carreras, en Negocios de la Madera, Ingeniería en Negocios y Gerencia y Desarrollo Social, debido al número de estudiantes se utilizó el universo, en las restantes cinco carreras se utilizó una muestra aleatoria probabilística, ver Tabla 1.

\section{Tabla 1}

Población de muestra de los participantes

\begin{tabular}{cccc}
\hline Universidad & Carrera & Población total & Participantes \\
\hline UNACIFOR & Ciencias Forestales & 328 & 177 \\
& Energía Renovable & 161 & 113 \\
& Negocios de la madera & 67 & 67 \\
\cline { 2 - 4 } & Ciencias Sociales & 132 & 98 \\
& Turismo & 277 & 161 \\
\cline { 2 - 4 } & Ungeniería en Negocios & 77 & 77 \\
& Gerencia y Desarrollo Social & 42 & 42 \\
\hline Total & & & 735 \\
\hline
\end{tabular}

Nota. Fuente: Elaboración propia. 
La muestra estuvo compuesta por 357 (48.57\%) estudiantes de la UNACIFOR, 259 (35.24\%) de la UPNFM y 119 (16.19\%) de la UMH, participaron 303 (41.22\%) hombres y 432 (58.78\%) mujeres. Las variables de contexto se midieron utilizando escalas ordinales, para el rendimiento académico se establecieron rangos, 70\% o menos (1), Entre 71 y $80 \%$ (2), Entre 81 y 90\% (3) y Entre 91 y 100\% (4), el nivel educativo se medió utilizando los niveles educativos de Doctorado (1), Maestría (2), Licenciatura (3), Secundaria completa (4), Primaria Completa (5) y Ninguno (6), los ingresos se midieron utilizando rangos, Menos de 5,000.00 (1), Entre L. 5,000.01 y 10,000.00 (2), Entre 10,000.01 y 15,000.00 (3), Entre 15,000.01 y 20,000.00 (4), Entre 20,000.01 y 25,000.00 (5), Entre 25,000.01 y 30,000.00 (6) y 30,000.01 o más (7).

Para el CC incorporado e institucionalizado se siguieron los planteamientos teóricos de diversos autores (Xu y Hampden-Thompson, 2012; Dumais, 2015), se elaboró un instrumento tipo escala Likert de cinco alternativas. Para medir el CC incorporado se consultó sobre las lecciones de arte, danza u otras formas de las artes, asistencia a museos, conciertos u otras representaciones y conducta de lectura, por ejemplo, "Visito museos de arte e historia, teatros, cines, casas de la cultura o explosiones de pintura y fotografía”. En el CC institucionalizado se midieron las credenciales educativas, por ejemplo, a). "Recibo premios/certificados por destacarme en el deporte, cultura o arte", b). "Tomo cursos sobre algún deporte (futbol, natación, tenis, ciclismo, etc.)", entre otros. Por la naturaleza del CC objetivado se utilizó una escala tipo nominal (Si/No), aquí se medió la posesión de recursos educativos en el hogar, la presencia de un lugar para estudiar, libros de referencia, un diccionario/enciclopedia en el hogar y accesorios tecnológicos, por ejemplo, “Tengo un espacio físico adecuado para estudiar en casa".

Se verificó la validez de contenido, constructo y la fiabilidad del instrumento, para la validez de contenido, se contó con apoyo de 3 expertos, quienes evaluaron el instrumentó a partir de una matriz que permitiera ofrecer observaciones en torno a la claridad y sencillez en la redacción de cada ítem; en la de constructo, la escala se sustenta en las teorías de Bourdeu (1997) quien plantea la existencia de las tres formas de CC, sobre la confiabilidad se utilizó el Alpha de Cronbach, en el capital Incorporado se observó un 0.804 y en el capital institucionalizado un 0.763 , ambos mayores a 0.70 . 
Tabla 2

Hipótesis nulas

\begin{tabular}{|c|c|c|}
\hline $\begin{array}{c}\text { Capital cultural } \\
\text { incorporado }\end{array}$ & $\begin{array}{c}\text { Capital cultural } \\
\text { institucionalizado }\end{array}$ & $\begin{array}{c}\text { Capital cultural } \\
\text { objetivado }\end{array}$ \\
\hline $\begin{array}{l}\text { Ho1: Los niveles de } \\
\text { CC incorporado de los } \\
\text { estudiantes no es distinto } \\
\text { según su rendimiento } \\
\text { académico }\end{array}$ & $\begin{array}{l}\text { Ho5: Los niveles de CC } \\
\text { institucionalizado de los } \\
\text { estudiantes no es distinto según } \\
\text { su rendimiento académico. }\end{array}$ & $\begin{array}{l}\text { Ho9: Los niveles de } \\
\text { CC objetivado de los } \\
\text { estudiantes no es distinto } \\
\text { según su rendimiento } \\
\text { académico. }\end{array}$ \\
\hline
\end{tabular}

Ho2: Los niveles de $\mathrm{CC}$ incorporado de los estudiantes no es distinto según el grado educativo del padre.

Ho3: Los niveles de CC incorporado de los estudiantes no es distinto según el grado educativo de la madre.
Ho6: Los niveles de CC institucionalizado de los estudiantes no es distinto según el grado educativo del padre.
Ho10: Los niveles de CC objetivado de los estudiantes no es distinto según el grado educativo del padre.
Ho7: Los niveles de CC institucionalizado de los estudiantes no es distinto según el grado educativo de la madre.
Ho11: Los niveles de CC objetivado de los estudiantes no es distinto según el grado educativo de la madre.
Ho3: Los niveles de CC incorporado de los estudiantes no es distinto según los ingresos.
Ho8: Los niveles de CC institucionalizado de los estudiantes no es distinto según los ingresos.
Ho12: Los niveles de CC objetivado de los estudiantes no es distinto según los ingresos.

Nota. Fuente: Elaboración propia.

\section{Plan de Análisis}

Se utilizó el programa SPSS versión 25, donde se transformaron y recodificaron las variables, seguidamente se calcularon las variables a partir de los ítems por escala. Respecto al análisis de los datos, el estudio incluye elementos de estadística descriptiva e inferencial, en esta última, a partir de los resultados de las pruebas de normalidad realizadas, se concluyó que para todas las variables los datos no provienen una población normal, atendiendo a este hallazgo, se utilizó la prueba estadística no paramétrica de Kruskal-Wallis para la prueba de hipótesis. 


\section{Resultados}

En esta primera sección se muestra el análisis descriptivo, se propuso una escala para comparar a nivel descriptivo cada una de las formas del CC entre los diferentes grupos, la cual se muestra en la Tabla 3.

\section{Tabla 3}

Escala propuesta para niveles descriptivos de los estados de CC

\begin{tabular}{ccc}
\hline Nivel & Media (Incorporado e Institucionalizado) & Media (Objetivado) \\
\hline Muy Alto & 4.21 a 5.00 & 0.81 a 1.00 \\
Alto & 3.41 a 4.20 & 0.61 a 0.80 \\
Medio & 2.61 a 3.40 & $0.41 \mathrm{a} 0.60$ \\
Bajo & 1.81 a 2.60 & 0.21 a 0.40 \\
Muy Bajo & 1.01 a 1.80 & 0.01 a 0.20 \\
\hline
\end{tabular}

Nota. Fuente: Elaboración propia.

En la tabla 4 se muestran los comparativos de las diferentes formas de CC entre las universidades, los datos reflejan que, por ejemplo, en el CC incorporado los estudiantes de la UPNFM muestran una $\mu=3.44$, mostrando un nivel "Alto" en función a la propuesta de la tabla 3 , la UNACIFOR y la UMH muestran niveles medios. En el CC institucionalizado se observó una $\mu=3.29$ en la UNACIFOR, superior a la observada en la UPNFM y la UMH, sin embargo, todas se encuentran en un nivel "Medio". Finalmente, en el CC objetivado se observó una $\mu=0.53$ en la UMH, pero las tres universidades muestran un nivel medio según la propuesta de la tabla 3.

\section{Tabla 4}

Niveles descriptivos de los estados de CC por universidad

\begin{tabular}{cccc}
\hline Universidad & $\boldsymbol{\mu}$ & & \\
\cline { 2 - 4 } & Incorporado & Institucionalizado & Objetivado \\
\hline UNACIFOR & 3.24 (DS:0.047) & 3.29 (DS:0.051) & 0.51 (DS:0.013) \\
UPNFM & 3.44 (DS:0.058) & 3.16 (DS:0.054) & 0.49 (DS:0.014) \\
UMH & 3.13 (DS:0.078) & $3.22(\mathrm{DS}: 0.091)$ & 0.53 (DS:0.022) \\
\hline
\end{tabular}

Nota. Fuente: Elaboración propia. 


\section{Tabla 5}

Descriptivos de los tres estados de CC

\begin{tabular}{cccl}
\hline Variable & Media & DS & Nivel \\
\hline Capital cultural incorporado & 3.29 & 0.91 & Medio \\
Capital cultural institucionalizado & 3.23 & 0.94 & Medio \\
Capital cultual objetivado & .51 & 0.24 & Medio \\
\hline
\end{tabular}

Nota. Fuente: Elaboración propia.

\section{Figura 2}

Diagrama de cajas y bigotes del CC incorporado ${ }^{3}$
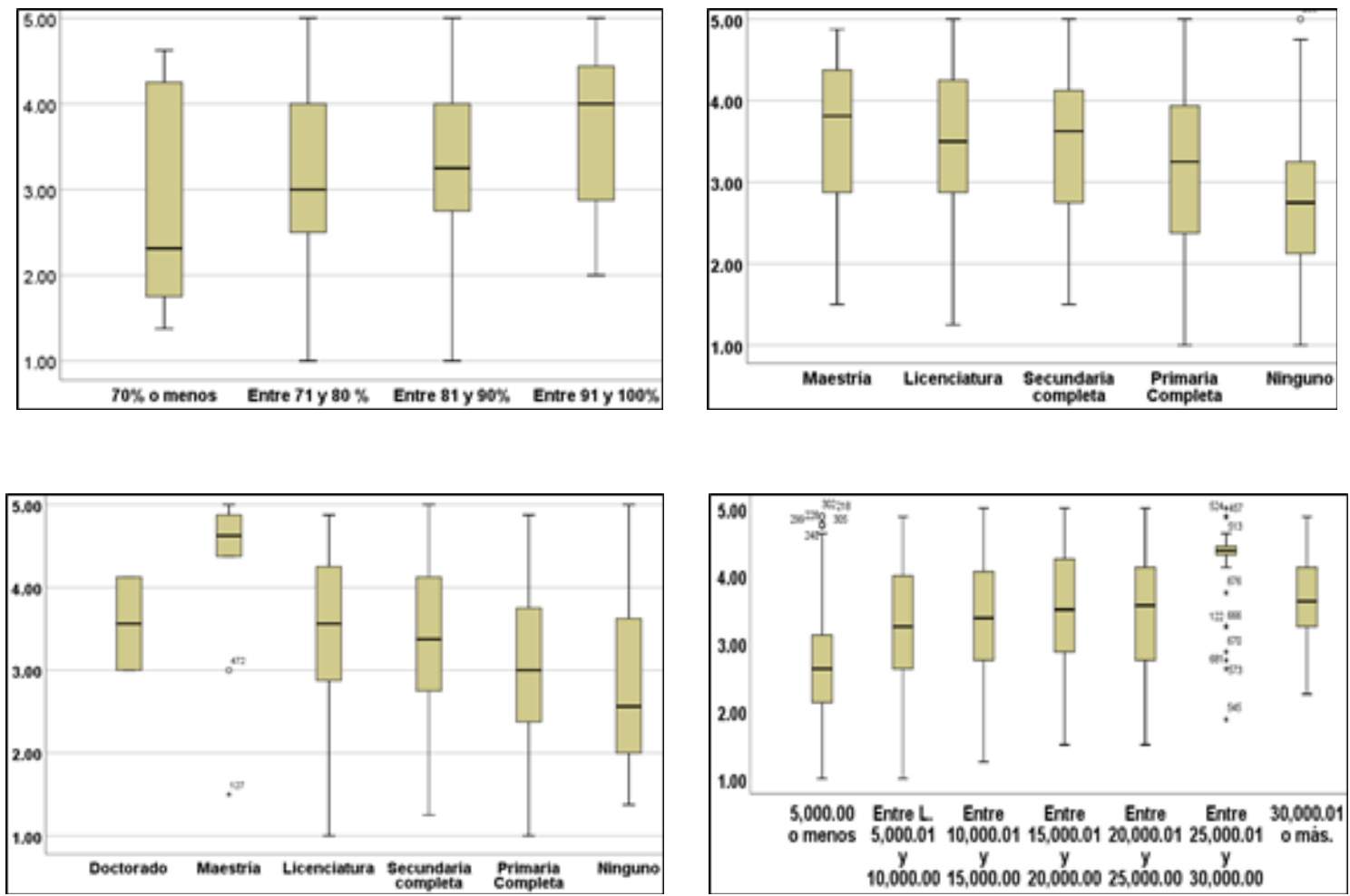

Nota. Fuente: Elaboración propia.

La Figura 2 muestra los diagramas de caja y bigotes del estado incorporado en los grupos de rendimiento académico, educación de los padres e ingresos, se observan $\mu$ mayores en los estudiantes de mayor rendimiento académico (2.74, 3.17, 3.33 y 3.74), mostrando un comportamiento ascendente, las $\mu$ del estado incorporado según la educación de los padres son de 2.82, 3.21, 3.46,

${ }^{3}$ Las figuras 2, 3 y 4 se deben leer en este orden horizontal; rendimiento académico, educación del padre, educación de la madre e ingresos. 
3.52, 3.58, el CC incorporado se incrementa a medida que aumenta el nivel educativo del padre . Con respecto a la educación de la madre, las $\mu$ observadas son de 2.81 (ninguna), 3.10 (primaria completa), 3.42 (secundaria completa), 3.51 (licenciatura), 4.15 (maestría), 3.56 (doctorado).

Las $\mu$ del estado incorporado entre los niveles de los ingresos familiares son de 2.76 (L 50,000.00), 3.27 (L 5,000.01 y 10,000.00), 3.41 (L 10,000.01 y 15,000.00), 3.46 (L 15,000.01 y 20,000.00), 3.47 (L. 20,000.01 y 25,000.00) 4.16 (L 25,000.01 y 30,000.00) y 3.61 (L 30,000.01 o más), los datos muestran que los niveles de CC incorporado son mayores en aquellos estudiantes cuyas familias tienen mayores ingresos (ver figura 2), mostrando un comportamiento ascendente.

\section{Figura 3}

Diagrama de cajas y bigotes del CC institucionalizado
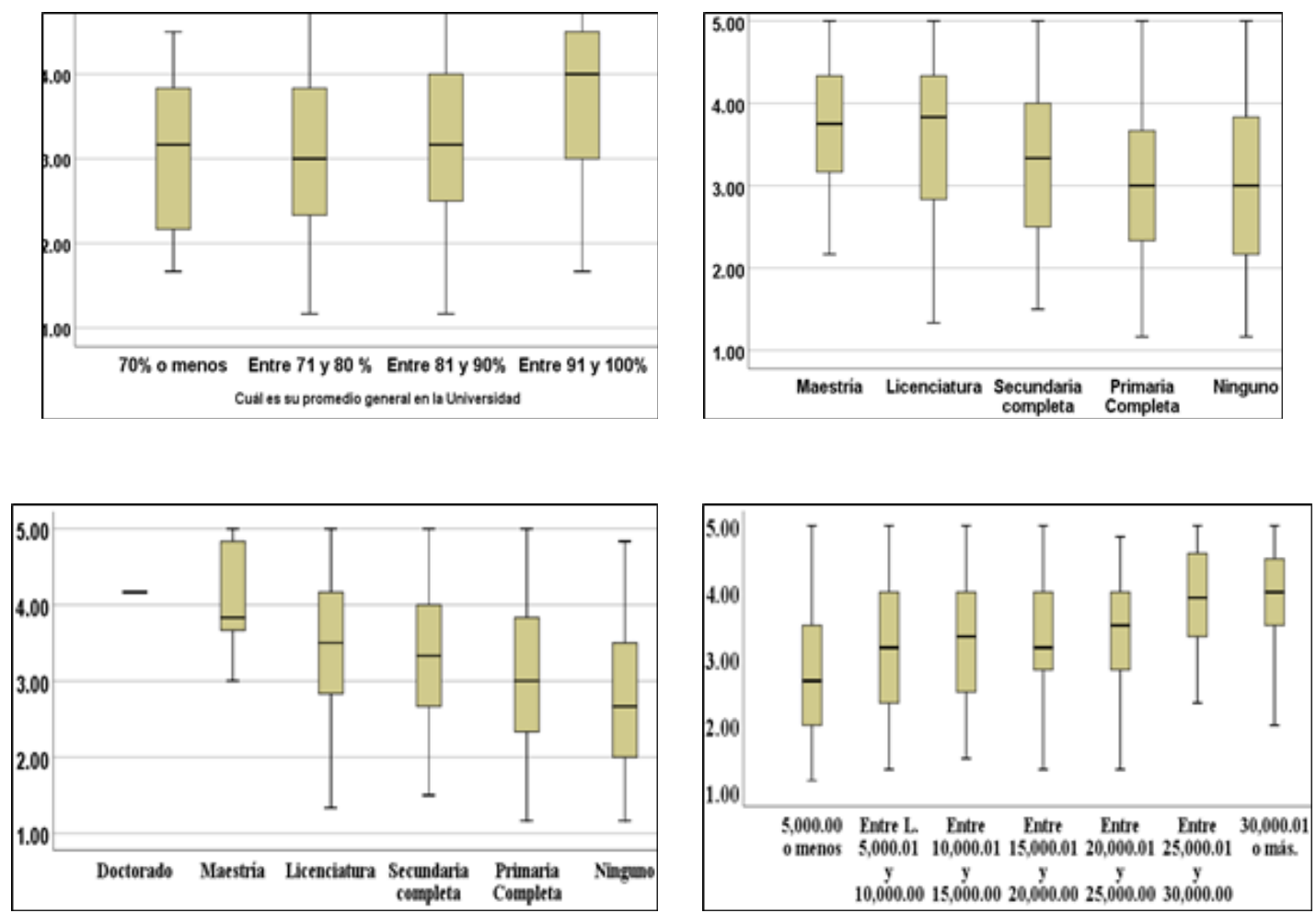

Nota. Fuente: Elaboración propia.

La Figura 3 muestra los diagramas de caja y bigotes del estado institucionalizado en los grupos de rendimiento académico, educación del padre, madre e ingresos, se observan $\mu$ mayores en los estudiantes de mayor rendimiento académico 3.08, 3.09, 3.25, 3.77, mostrando un comportamiento ascendente, las $\mu$ del estado institucionalizado según la educación de los padres son de $3.01,3.01,3.28,3.58,3.71$, se observa que el CC institucionalizado se incrementa 
a medida que aumenta el nivel educativo del padre. Con respecto a la educación de la madre, las $\mu$ observadas son de 2.79 (ninguna), 3.05 (primaria completa), 3.32 (secundaria completa), 3.48 (licenciatura), 4.07 (maestría), 4.17 (doctorado).

Las $\mu$ del estado institucionalizado entre los niveles de los ingresos familiares son de 2.80 (L menos de 50,000.00), 3.17 (L 5,000.01 y 10,000.00), 3.30 (L 10,000.01 y 15,000.00), 3.33 (L $15,000.01$ y 20,000.00), 3.45 (L. 20,000.01 y 25,000.00) 4.87 (L 25,000.01 y 30,000.00) у 3.85 (L 30,000.01 o más), los datos muestran que los niveles de CC institucionalizado son mayores en aquellos estudiantes cuyas familias tienen mayores ingresos (ver figura 3), mostrando un comportamiento ascendente.

\section{Figura 4}

Diagrama de cajas y bigotes del CC objetivado
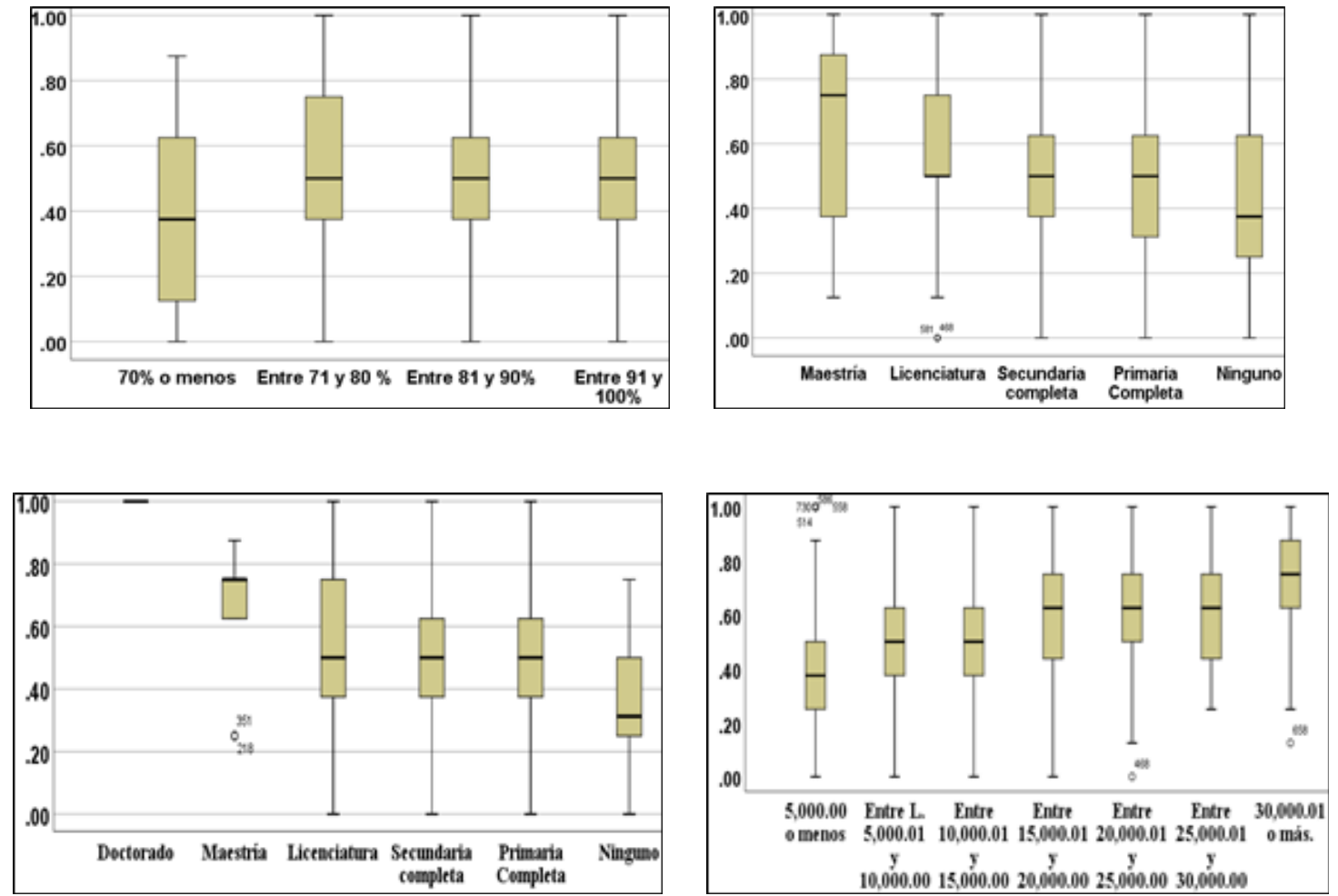

Nota. Fuente: Elaboración propia

La Figura 4 muestra los diagramas de caja y bigotes del estado objetivado en los grupos de rendimiento académico, educación del padre, de la madre e ingresos, se observan $\mu 0.40,0.51,0.51$, 0.51, llama especial atención que los estudiantes que tienen bajo rendimiento académico muestran bajo CC objetivado, las $\mu$ del estado objetivado según la educación de los padres son de $0.39,0.48$, 
0.54, 0.58 y 0.68, se observa se incrementa a medida que aumenta el nivel educativo del padre. Con respecto a la educación de la madre, las $\mu$ observadas son de 0.33 (ninguna), 0.49 (primaria completa), 0.52 (secundaria completa), 0.57 (licenciatura), 0.67 (maestría), 1.00 (doctorado).

Las $\mu$ del estado objetivado entre los niveles de los ingresos familiares son de 0.40 (L menos de 50,000.00), 0.47 (L 5,000.01 y 10,000.00), 0.53 (L 10,000.01 y 15,000.00), 0.59 (L 15,000.01 y 20,000.00), 0.57 (L. 20,000.01 y 25,000.00) 0.59 (L 25,000.01 y 30,000.00) y 0.69 (L 30,000.01 o más), los datos muestran que los niveles de CC objetivado son mayores en aquellos estudiantes cuyas familias tienen mayores ingresos (ver Figura 4).

\section{Comprobación de Hipótesis}

Los datos descriptivos expuestos en la sección anterior dejan en evidencia que los niveles de CC en sus tres formas son diferentes según el rendimiento académico de los estudiantes, la educación de sus padres y los ingresos, sin embargo, para encontrar significancia estadística, a continuación, se muestran las pruebas de hipótesis.

\section{Comparaciones con el Rendimiento Académico}

La prueba estadística de Kruskal-Wallis para la diferencia de medianas del CC incorporado en los estudiantes entre los grupos de rendimientos académicos mostró un p-valor de $0.000<\alpha: 0.05$, por tanto, se rechaza la H01 (ver Tabla 2) y se concluye que existe evidencia estadísticamente significativa en el CC incorporado, en al menos un grupo de rendimiento académico observado, la prueba post hoc para diferencias de medianas entre los grupos mostraron p-valores $<\alpha: 0.05$ (ver Tabla 6).

\section{Tabla 6}

Prueba post hoc del CC incorporado y el de rendimiento académico

\begin{tabular}{cc}
\hline Grupos & p-valores \\
\hline $70 \%$ o menos entre 91 y $100 \%$ & 0.000 \\
Entre 71 y $80 \%$ entre 91 y $100 \%$ & 0.000 \\
Entre 81 y $90 \%$ entre 91 y $100 \%$ & 0.004 \\
\hline
\end{tabular}

Nota. Fuente: Elaboración propia

La prueba estadística de Kruskal-Wallis para la diferencia de medianas del CC institucionalizado en los estudiantes entre los grupos de rendimientos académicos mostró un p-valor de $0.000<\alpha: 0.05$, por tanto, se rechaza la H05 (ver Tabla 2) y se concluye que existe evidencia estadísticamente significativa 
en el CC institucionalizado, en al menos un grupo de rendimiento académico observado, la prueba post hoc para diferencias de medianas entre los grupos mostraron p-valores $<\alpha: 0.05$ (ver Tabla 7).

\section{Tabla 7}

Prueba post hoc del CC institucionalizado y el de rendimiento académico

\begin{tabular}{cc}
\hline Grupos & p-valores \\
\hline $70 \%$ o menos entre 71 y $80 \%$ & 0.016 \\
71 y $80 \%$ entre 91 y $100 \%$ & 0.000 \\
81 y $90 \%$ entre 91 y $100 \%$ & 0.000 \\
\hline
\end{tabular}

Nota. Fuente: Elaboración propia

Sin embargo, la prueba estadística de Kruskal-Wallis para la diferencia de medianas del CC objetivado en los estudiantes entre los grupos de rendimientos académicos mostró un p-valor de 0.287> $>$ :0.05, por tanto, se acepta la H09 (ver Tabla 2) y se concluye que no existe evidencia estadísticamente significativa en el CC objetivado, en al menos un grupo de rendimiento académico observado.

\section{Comparaciones con la Educación de sus Padres}

La prueba estadística de Kruskal-Wallis para la diferencia de medianas del CC incorporado en los estudiantes entre los grupos de grado educativo del padre mostró un p-valor de $0.000<\alpha: 0.05$, por tanto, se rechaza la H02 (ver Tabla 2) y se concluye que existe evidencia estadísticamente significativa en el CC incorporado, en al menos un grupo del grado educativo del padre observado, la prueba post hoc para diferencias de medianas entre los grupos mostraron $p$-valores $<\alpha: 0.05$ (ver Tabla 8).

\section{Tabla 8}

Prueba post hoc del CC incorporado y el grado educativo del padre

\begin{tabular}{cc}
\hline Grupos & p-valores \\
\hline Ninguno y primaria completa & 0.002 \\
Ninguno y secundaria completa & 0.000 \\
Ninguno y Licenciatura & 0.000 \\
Ninguno y Maestría & 0.001 \\
Primaria completa y Licenciatura & 0.022 \\
\hline
\end{tabular}

Nota. Fuente: Elaboración propia. 
Para la diferencia de medianas del CC incorporado en los estudiantes entre los grupos de grado educativo de la madre mostró un p-valor de $0.000<\alpha: 0.05$, por tanto, se rechaza la H03 (ver Tabla 2) y se concluye que existe evidencia estadísticamente significativa en el CC incorporado, en al menos un grupo del grado educativo de la madre observado, la prueba post hoc para diferencias de medianas entre los grupos mostraron $p$-valores $<\alpha: 0.05$ (ver Tabla 9).

\section{Tabla 9}

Prueba post hoc del CC incorporado y el grado educativo de la madre

\begin{tabular}{cc}
\hline Grupos & p-valores \\
\hline Ninguno y secundaria completa & 0.000 \\
Ninguno y Licenciatura & 0.000 \\
Ninguno y doctorado & 0.000 \\
Primaria completa y secundaria completa & 0.001 \\
Primaria completa y Licenciatura & 0.000 \\
Primaria completa y doctorado & 0.008 \\
\hline
\end{tabular}

Nota. Fuente: Elaboración propia

La prueba estadística de Kruskal-Wallis para la diferencia de medianas del CC institucionalizado en los estudiantes entre los grupos de grado educativo de los padres mostró un p-valor de 0.001<a:0.05, por tanto, se rechaza la H06 (ver Tabla 2) y se concluye que existe evidencia estadísticamente significativa en el CC institucionalizado, en al menos un grupo del grado educativo de los padres observado, la prueba post hoc para diferencias de medianas entre los grupos mostraron p-valores $<\alpha: 0.05$ (ver Tabla 10).

Para la diferencia de medianas del CC institucionalizado en los estudiantes entre los grupos de grado educativo de las madres mostró un p-valor de $0.000<\alpha: 0.05$, por tanto, se rechaza la H07 (ver Tabla 2) y se concluye que existe evidencia estadísticamente significativa en el CC institucionalizado, en al menos un grupo del grado educativo de las madres observado, la prueba post hoc para diferencias de medianas entre los grupos mostraron p-valores $<\alpha: 0.05$ (ver tabla 11). 


\section{Tabla 10}

Prueba post hoc del CC institucionalizado y el grado educativo de padres

\begin{tabular}{cc}
\hline Grupos & p-valores \\
\hline Primaria completa y secundaria & 0.031 \\
Primaria completa y licenciatura & 0.000 \\
Primaria completa y maestría & 0.003 \\
Ninguno licenciatura & 0.000 \\
Ninguno maestría & 0.007 \\
Secundaria completa y licenciatura & 0.027 \\
\hline
\end{tabular}

Nota. Fuente: Elaboración propia.

\section{Tabla 11}

Prueba post hoc del CC institucionalizado y el grado educativo de las madres

\begin{tabular}{cc}
\hline Grupos & p-valores \\
\hline Ninguno y secundaria completa & 0.000 \\
Ninguno y licenciatura & 0.000 \\
Ninguno maestría & 0.000 \\
Primaria completa y secundaria & 0.001 \\
Primaria completa y licenciatura & 0.000 \\
Primaria completa y maestría & 0.008 \\
\hline
\end{tabular}

Nota. Fuente: Elaboración propia.

Entre tanto, la prueba de Kruskal-Wallis para la diferencia de medianas del CC objetivado en los estudiantes entre los grupos de grado educativo de los padres mostró un p-valor de $0.000<\alpha: 0.05$, por tanto, se rechaza la H10 (ver Tabla 2) y se concluye que existe evidencia estadísticamente significativa en el CC objetivado, en al menos un grupo del grado educativo de los padres observado, la prueba post hoc para diferencias de medianas entre los grupos mostraron p-valores $<\alpha: 0.05$ (ver Tabla 12). 


\section{Tabla 12}

Prueba post hoc del CC objetivado y el grado educativo de los padres

\begin{tabular}{cc}
\hline Grupos & p-valores \\
\hline Ninguno y primaria completa & 0.008 \\
Ninguno y secundaria completa & 0.000 \\
Ninguno y licenciatura & 0.000 \\
Ninguno y maestría & 0.000 \\
Primaria completa y licenciatura & 0.004 \\
Primaria completa y maestría & 0.002 \\
\hline
\end{tabular}

Nota. Fuente: Elaboración propia.

Finalmente, la prueba de Kruskal-Wallis para la diferencia de medianas del CC objetivado en los estudiantes entre los grupos de grado educativo de las madres mostró un p-valor de $0.000<\alpha: 0.05$, por tanto, se rechaza la H011 (ver Tabla 2) y se concluye que existe evidencia estadísticamente significativa en el CC objetivado, en al menos un grupo del grado educativo de la madre observado, la prueba post hoc para diferencias de medianas entre los grupos mostraron $p$-valores $<\alpha: 0.05$ (ver Tabla 13).

\section{Tabla 13}

Prueba post hoc del CC objetivado y el grado educativo de los padres

\begin{tabular}{cc}
\hline Grupos & p-valores \\
\hline Ninguno y primaria completa & 0.000 \\
Ninguno y secundaria completa & 0.000 \\
Ninguno y licenciatura & 0.000 \\
Ninguno y maestría & 0.000 \\
\hline
\end{tabular}

Nota. Fuente: Elaboración propia. 


\section{Comparaciones con el Ingreso}

La prueba estadística de Kruskal-Wallis para la diferencia de medianas del CC incorporado en los estudiantes entre los grupos de ingresos mostró un p-valor de $0.000<\alpha: 0.05$, por tanto, se rechaza la H04 (ver Tabla 2) y se concluye que existe evidencia estadísticamente significativa en el CC incorporado, en al menos un grupo de ingresos observado, la prueba post hoc para diferencias de medianas entre los grupos mostraron p-valores $<\alpha: 0.05$ (ver Tabla 14).

\section{Tabla 14}

Prueba post hoc del CC incorporado y el ingreso

\begin{tabular}{cc}
\hline Grupos & p-valores \\
\hline $5,000.00$ o menos entre $5,000.01$ y $10,000.00$ & 0.000 \\
$5,000.00$ o menos entre $10,000.01$ y $15,000.00$ & 0.000 \\
$5,000.00$ o menos entre $15,000.01$ y $20,000.00$ & 0.000 \\
$5,000.00$ o menos entre 20,000.01 y $25,000.00$ & 0.000 \\
$5,000.00$ o menos entre $30,000.0$ o más. & 0.000 \\
$5,000.00$ o menos entre $25,000.01$ y $30,000.00$ & 0.000 \\
$5,000.01$ y $10,000.00$ entre $25,000.01$ y $30,000.00$ & 0.000 \\
$10,000.01$ y $15,000.00$ entre $25,000.01$ y $30,000.00$ & 0.000 \\
$15,000.01$ y 20,000.00 entre $25,000.01$ y $30,000.00$ & 0.002 \\
$20,000.01$ y 25,000.00 entre 25,000.01 y 30,000.00 & 0.006 \\
\hline
\end{tabular}

Nota. Fuente: Elaboración propia.

Entre tanto, la diferencia de medianas del CC institucionalizado en los estudiantes entre los grupos de ingresos mostró un p-valor de 0.000<a:0.05, por tanto, se rechaza la H08 (ver Tabla 2) y se concluye que existe evidencia estadísticamente significativa en el CC institucionalizado, en al menos un grupo de ingresos observado, la prueba post hoc para diferencias de medianas entre los grupos mostraron $\mathrm{p}$-valores $<\alpha: 0.05$ (ver Tabla 15). 


\section{Tabla 15}

Prueba post hoc del CC institucionalizado y el ingreso

\begin{tabular}{cc}
\hline Grupos & p-valores \\
\hline $5,000.00$ o menos entre $5,000.01$ y $10,000.00$ & 0.006 \\
$5,000.00$ o menos entre $10,000.01$ y $15,000.00$ & 0.000 \\
$5,000.00$ o menos entre $15,000.01$ y $20,000.00$ & 0.001 \\
$5,000.00$ o menos entre 20,000.01 y 25,000.00 & 0.000 \\
$5,000.00$ o menos entre 25,000.01 y 30,000.00 & 0.000 \\
$5,000.00$ o menos entre 30,000.01 o más & 0.000 \\
$5,000.01$ y 10,000.00 entre 25,000.01 y 30,000.00 & 0.001 \\
$5,000.01$ y 10,000.00 entre 30,000.01 o más & 0.001 \\
$10,000.01$ y 15,000.00 entre 25,000.01 y 30,000.00 & 0.026 \\
$10,000.01$ y 15,000.00 entre 30,000.01 o más & 0.022 \\
\hline
\end{tabular}

Nota. Fuente: Elaboración propia.

Entre tanto, era previsible, la diferencia de medianas del CC objetivado en los estudiantes entre los grupos de ingresos mostró un p-valor de $0.000<\alpha: 0.05$, por tanto, se rechaza la H12 (ver Tabla 2) y se concluye que existe evidencia estadísticamente significativa en el CC objetivado, en al menos un grupo de ingresos observado, la prueba post hoc para diferencias de medianas entre los grupos mostraron p-valores $<\alpha: 0.05$ (ver Tabla 16).

\section{Tabla 16}

Prueba post hoc del CC objetivado y el ingreso

\begin{tabular}{cc}
\hline Grupos & p-valores \\
\hline $5,000.00$ o menos entre $5,000.01$ y $10,000.00$ & 0.020 \\
$5,000.00$ o menos entre $10,000.01$ y $15,000.00$ & 0.000 \\
$5,000.00$ o menos entre $15,000.01$ y $20,000.00$ & 0.000 \\
$5,000.00$ o menos entre 20,000.01 y 25,000.00 & 0.000 \\
$5,000.00$ o menos entre 25,000.01 y 30,000.00 & 0.000 \\
$5,000.00$ o menos entre 30,000.01 o más & 0.000 \\
$5,000.01$ y 10,000.00 entre $15,000.01$ y 20,000.00 & 0.011 \\
$5,000.01$ y 10,000.00 entre 30,000.01 o más & 0.000 \\
$10,000.01$ y 15,000.00 entre 30,000.01 o más & 0.004 \\
\hline
\end{tabular}

Nota. Fuente: Elaboración propia. 


\section{Discusión de Resultados}

Diversos autores han analizado el vínculo entre el CC y el rendimiento académico de estudiantes (Evans et al., 2010; Xu y Hampden-Thompson, 2012; Yamamoto y Brinton, 2010), los hallazgos de esta investigación son congruentes con estos estudios debido a que se observó que los estudiantes que muestran mayores niveles de capital cultural, en dos de sus tres estados (Institucionalizado y objetivado), obtienen un mayor rendimiento académico.

Aunado a lo anterior, estos hallazgos respaldan la teoría de Bourdieu (1973) la cual sugiere que la orientación de un estudiante se desarrolla en relación con la cantidad de CC que posee; un estudiante de bajo nivel socioeconómico es consciente que las personas de esa clase tienden a tener bajo $\mathrm{CC}$ y que, sin éste, se reducen las probabilidades de tener éxito educativo. Por lo tanto, los estudiantes universitarios de bajo nivel socioeconómico tenderán, en promedio, a tener expectativas más bajas sobre el éxito educativo en la universidad y es menos probable que utilicen el poco CC que tienen porque no ven muchas posibilidades de tener éxito académico.

Los resultados son coherentes con Jæger (2011) quien encontró efectos del CC en el rendimiento de los estudiantes en lectura y matemáticas en los Estados Unidos, con indicadores que reflejan la cultura intelectual y los hábitos de lectura como los más importantes para los estudiantes. Igualmente, coinciden con Roksa y Potter (2011) quienes atribuyen parte de la relación entre el CC y el rendimiento de los estudiantes en los Estados Unidos a las prácticas de crianza y al papel importante de inversión de los padres en educación.

Sobre los resultados encontrados con la educación de los padres, éstos sugieren que los antecedentes educativos familiares tienen un impacto importante en el logro educativo de los estudiantes universitarios, así, los niveles de educación alcanzados por los padres afectan positivamente la probabilidad que un estudiante tenga éxito en la universidad.

Desde la perspectiva de la educación de los padres, De Graaf, De Graaf y Krayykamp (2000) afirman que los padres que leen frecuentemente tienen habilidades cognitivas, lingüísticas y lectoras que pueden transmitir a sus hijos, y proporcionan un ambiente de aprendizaje estimulante que actúa como ejemplo. Los resultados del presente estudio son congruentes con otros estudios que indican que los padres con niveles educativos altos muestran mayor interés en el desempeño académico de sus hijos (Jiménez, 2008), así, el nivel educativo modifica las creencias y los estilos de comportamiento de los padres hacia sus hijos y por tanto influyen en el rendimiento académico. 
Coindicen con diversos planteamientos que sugieren que el CC institucional de los padres es un "determinante de las expectativas y los logros educativos y ocupacionales" (Lehmann, 2007, p. 90), los resultados muestran que los estudiantes cuyos padres no han asistido a instituciones postsecundarias pueden encontrar el entorno más intimidante y menos familiar que los estudiantes cuyos padres han asistido a instituciones postsecundarias.

En torno a la educación de los padres como elemento de trasmisión de CC, si bien las familias pueden ayudar a facilitar la transición de los estudiantes a instituciones postsecundarias utilizando diversas formas de capital, en general, muchos estudiantes se encuentran en desventaja académica en comparación con los estudiantes cuyos padres tienen un grado educativo (Lehmann, 2007). Las expectativas educativas de los padres con sus hijos constituye un predictor clave de las aspiraciones educativas (Wu y Bai, 2015). Por ejemplo, Lyons (2006) encontró que las actitudes de los padres hacia las calificaciones educativas y el estímulo, eran factores importantes relacionados con las decisiones de los estudiantes. De acuerdo con Moakler y Kim (2014), los hallazgos de esta investigación dan cuanta que los padres altamente educados tienen más probabilidades de cumplir los objetivos de obtener calificaciones terciarias en sus hijos.

Desde esta perspectiva, los resultados ponen de manifiesto que los padres con educación universitaria pueden tener más probabilidades de participar en la orientación de los jóvenes en el proceso de construcción del CC que es valorado por las instituciones educativas, desde esta mirada, aquellos padres con un nivel socioeconómico más alto pueden tener mayores expectativas educativas de sus hijos (Carolan y Wasserman, 2015), además pueden estar más involucrados en su educación (Cheadle y Amato, 2011).

Estos hechos, advierten que el papel de la familia va más allá de las formas típicas de capital social, debido a que la familia proporciona el contexto en donde los estudiantes desarrollan su identidad. Por esta razón, los factores relacionados con la familia están fuertemente ligados al concepto de habitus de Bourdieu.

Sobre los ingresos económicos y su papel en el CC, los resultados de esta investigación son coherentes con investigaciones anteriores que han demostrado que el ingreso y la riqueza de la familia son grandes predictores del éxito educativo (Shapiro et al., 2013), por ejemplo, Gibb et al. (2012) sugiere que el ingreso familiar es un fuerte predictor del logro educativo en la edad adulta. Como señala Bourdieu (1986), el valor del capital económico proviene de su valor para generar un cambio. Cuando eso ocurre, es probable que los estudiantes de familias 
económicamente adineradas puedan pagar libros, computadoras portátiles y otras ayudas para estudiar. Al pagar por estos objetos, los estudiantes intercambian capital económico por activos no financieros como el CC.

Alineado a Bourdieu y Passeron (1970,1990), los hallazgos sugieren que los padres con un nivel socioeconómico alto equipan a sus hijos con $\mathrm{CC}$, que se traduce en habilidades lingüísticas, sociales y culturales. Esta dinámica puede explicarse, en tanto que, las instituciones educativas requieren la posesión de estas habilidades para lograr el éxito educativo y no pueden a través de la enseñanza transmitir estas competencias a los jóvenes que tienen bajo niéveles socioeconómicos.

Los resultados de la investigación apoyan la teoría de Bourdieu (1973), en el sentido que las universidades reproducen la desigualdad social, así, los jóvenes de entornos de bajo nivel socioeconómico no están expuestos a un entorno necesario para construir CC y se encuentran en desventaja cuando no muestran el habitus requerido por las IES. Este proceso conduce finalmente a la reproducción de las desigualdades generadas por el nivel socioeconómico, porque recompensan las manifestaciones de la cultura dominante que se traducen en niveles más altos de logros educativos.

Finamente, estos resultados parecen distanciarse de la propuesta de Sen (2000) y su teoría de las "capacidades", por cuanto dejan en evidencia que el Estado, representado por las IES, no genera igualdad en las capacidades de los jóvenes, centra su atención en ampliar las oportunidades, pero al hacerlo, trata a todos por igual, dejando en evidencia un trato desigual para los jóvenes que provienen de contextos más desfavorables, como lo sostiene el autor, igualdad en medios y recursos no implica una igualdad en libertad para que los jóvenes se desempeñen de forma similar en su paso por el sistema educativo superior.

\section{Conclusiones}

Se concluye que, aquellos estudiantes que tienen un mejor rendimiento académico muestran distintos niveles de capital de capital cultural incorporado e institucionalizado sustentado en las pruebas de KruskalWallis con $p$-valores de $0.000<\alpha=0.05$, dejando en evidencia que estudiantes que poseen mejor rendimiento académico, primero, poseen un mayor capital incorporado, es decir, son los que tienen encarnado símbolos de una cultura dominante que han adquirido producto de socializar con patrones culturales que principalmente están en el núcleo de la familia (capital incorporado), segundo, son estudiantes que valoran las credenciales educativas y reconocen el valor de la posesión de títulos profesionales, calificaciones académicas, diplomas y reconocimiento adquiridos que son socialmente reconocibles en los que tienen un mayor éxito educativo (capital institucionalizado). Con p-valores de $0.000>\alpha=0.05$, en 
los niveles de CC objetivado, no se observaron diferencia en los grupos de rendimiento académico, esto permite concluir que poseer recursos en el hogar, entre ellos, material de lectura, espacios físicos que facilitan el aprendizaje; elementos culturales, por ejemplo, literatura, esculturas, música y obras de arte, no parece tener incidencia en el rendimiento académico de los estudiantes.

Las pruebas estadísticas en los grupos de educación de los padres, con p- valores de $0.000<\alpha=0.05$, concluyen que el contexto familiar juega un papel importante para incorporar el CC a los hijos, el estudio concluye que los estudiantes cuyos padres de familia tienen un mayor grado educativo transmiten ese conjunto de símbolos culturales, así, los estudiantes cuyos padres tienen una mejor certificación académica tiende a mostrar altos niveles de capital y valoran el conjunto de símbolos culturales que se requieren para ser éxito en la universidad.

Sobre el ingreso económico de los padres, con $\mathrm{p}$ - valores de $0.000<\alpha=0.05$, se concluye que los recursos económicos, capital económico llamado por Bourdieu (1997), continúa siendo un aspecto fundamental para que los estudiantes universitarios incorporen los valores culturales que son importantes en el contexto de la universidad, el estudio deja en evidencia que los estudiantes cuyos ingresos familiares son bajos muestran bajos niveles en las tres formas de capital o viceversa.

El limitado acceso a recursos económicos de las familias genera restricciones para asignar valor a las actividades culturales, entendiendo que todas ellas implican invertir recursos económicos que no poseen. Sobre el CC institucionalizado es evidente que hoy en día certificar o acreditar las habilidades de los hijos implica disponer de recursos, bien para seguir en la universidad o para matricular a sus hijos en cursos especializados.

\section{Recomendaciones}

Centrado en los hallazgos de la investigación, surgen un conjunto de recomendaciones que se deben tomar consideración para fortalecer el CC en las universidades, en primer lugar, se debe favorecer los espacios de fortalecimiento a la cultura y orientar acciones que compensen las desigualdades culturales que muestran los estudiantes que provienen de niveles socioeconómicos bajos, en consecuencia, actividades como la música, las artes, la literatura deben constituirse en un espacio transversal en la formación de los estudiantes universitarios. 
En segundo lugar, las instituciones de educación superior juegan un papel importante para fortalecer CC objetivado, orientando recursos de su presupuesto para ofrecer, por ejemplo, espacios físicos adecuados para que los estudiantes pueden realizar sus labores académicas, incrementar el número de equipos computacionales para permitir a los estudiantes acceder a recursos que no tienen acceso en sus hogares producto del acceso limitado a los recursos, así como, facilitar espacios de formación para los estudiantes que les permita certificar diferentes competencias que han adquirido durante su formación, más allá de concederles un título, esto implica crear cursos especializados en diversas áreas que conlleven a certificar sus habilidades.

Con énfasis en los hallazgos, en tercer lugar, se debe tomar en consideración los niveles de $\mathrm{CC}$ en las pruebas de admisión para tener en cuenta los diferentes niveles de competencia cultural que poseen los estudiantes, generar oportunidades para que todos tengan posibilidades de acceder es importante, sin embargo, el trato que recibe el estudiante una vez que ha ingresado al sistema para compensar esas desigualdades y generar las condiciones para fortalecer el CC es determinante en el éxito o fracaso del estudiante en el sistema.

\section{Referencias Bibliográficas}

Archer, L., DeWitt, J., Osborne, J., Dillon, J., Willis, B., y Wong, B. (2012). Science aspirations, capital, and family habitus: how families shape children's engagement and identification with science. Am. Educ. Res. J. 49, 881-908. doi: 10.3102/0002831211433290

Bourdieu, P. (1973). Cultural reproduction and social reproduction, in Knowledge, Education, and Cultural Change: Papers in the Sociology of Education (ed. R. Brown), Tavistock Publications, London, pp. 71-112.

Bourdieu, P. (1979). “Los Tres Estados del Capital Cultural”, en Sociológica, UAM-Azcapotzalco, México, núm 5, pp. 11-17. 1.

Bourdieu, P. (1984). Distinction: A Social Critique of the Judgement of Taste. Translated by Richard Nice. Cambridge: Harvard University Press.

Bourdieu, P. (1987). Los tres estados del capital cultural. Sociológica, 5, 11-17. Recuperado de http:// www.sociologicamexico.azc.uam.mx/index.php/Sociologica/article/view/1043 
Bourdieu, P., (1986). The forms of capital. In: Richardson, J.G. (Ed.), Handbook of Theory and Research in the Sociology of Education. Greenwood Press, New York, pp. 241-258.

Bourdieu, P., Passeron, J.-C., 1990. Reproduction in Education, Society and Culture. Sage, London.

Bourdieu, P., y Wacquant, L. J. (1992). An invitation to Reflexive Sociology. Chicago, IL: University of Chicago Press.

Bourdieu, Pierre, 1997. The forms of capital. In: Halsey, A.H., Lauder, Hugh, Brown, Phillip, Stuart Wells, Amy (Eds.), Education: Culture, Economy, and Society. Oxford University Press, Oxford, England, pp. 46-58.

Bourdieu, Pierre, Passeron, Jean-Claude, (1977). Reproduction in Education, Society and Culture. Sage, Beverly Hills, CA.

Breinholt, A., \& Jæger, M. M. (2020). How does cultural capital affect educational performance: Signals or skills? The British Journal of Sociology, 71(1), 28-46. doi:10.1111/1468-4446.12711

Carolan, B. V., and Wasserman, S. J. (2015). Does parenting style matter? concerted cultivation, educational expectations, and the transmission of educational advantage. Sociol. Perspect. 58, 168-186. doi: 10.1177/0731121414562967

Castillo, G., M. (2018). El capital cultural familiar en la educación preuniversitaria Estrategias familiares de estudiantes del Colegio Central Universitario Mariano Moreno San Juan, Argentina

Cheadle, J. E., y Amato, P. R. (2011). A quantitative assessment of lareau's qualitative conclusions about class, race, and parenting. J. Fam. Issues 32, 679-706. doi: 10.1177/0192513X10386305

Cheng Yong Tan, Meiyan Lyu \& Baiwen Peng (2020) Academic Benefits from Parental Involvement are Stratified by Parental Socioeconomic Status: A Meta-analysis, Parenting, 20:4, 241-287, DOI: $10.1080 / 15295192.2019 .1694836$

Cheng, S.-T., y Kaplowitz, S. A. (2016). Family economic status, cultural capital, and academic achievement: The case of Taiwan. International Journal of Educational Development, 49, 271-278. doi:10.1016/j.ijedudev.2016.04.002

De Graaf, N. D., De Graaf, P. M., y Kraaykamp, G. (2000). Parental cultural capital and educational attainment in the Netherlands: A refinement in the cultural capital perspective. Sociology of Education, 73, 92-111.

DiMaggio, P. (1982). Cultural capital and school success: The impact of status culture participation on the grades of U.S. high school students. American Sociological Review, 47, 189-201. 
Dumais, S. A. (2015). Cultural Capital and Education. International Encyclopedia of the Social y Behavioral Sciences, 375-381. doi:10.1016/b978-0-08-097086-8.10433-7

Evans, M. D. R., Kelley, J., Sikora, J., \& Treiman, D. J. (2010). Family scholarly culture and educational success: Books and schooling in 27 nations. Research in Social Stratification and Mobility, 28(2), 171-197. doi:10.1016/j.rssm.2010.01.002

Gibb, S. J., Fergusson, D. M., and Horwood, L. J. (2012). Childhood family income and life outcomes in adulthood: findings from a 30-year longitudinal study in new zealand. Soc. Sci. Med. 74, 1979-1986. doi: 10.1016/j.socscimed.2012.02.028

Jæger, M. M. (2011). Does Cultural Capital Really Affect Academic Achievement? New Evidence from Combined Sibling and Panel Data. Sociology of Education, 84(4), 281-298. doi: $10.1177 / 0038040711417010$

Jiménez. M. (2008). El papel de las madres en la motivación que presentan sus hijos hacia el aprendizaje escolar. Tesis de Doctorado no publicada: Universidad Nacional Autónoma de México.

Klimczuk, A. (2015). Cultural Capital. The Wiley Blackwell. Encyclopedia of Consumption and Consumer Studies, 1-3. doi:10.1002/9781118989463.wbeccs083

Košutić, I., (2017). The Role of Cultural Capital in Higher Education Access and Institutional Choice. Institute for Social Research, Zagreb, 7(1), 149-196.

Lareau, A. (2011). Unequal Childhoods: Class, Race, and Family Life, Second Edition with an Update a Decade Later, University of California Press, Berkeley.

Lehmann, W. (2007). "I just didn't feel like I fit in": The role of habitus in university dropout decisions. Canadian Journal of Higher Education, 37(2), 89-110.

Lyons, T. (2006). "Choosing physical science courses: the importance of cultural and social capital in the enrolment decisions of high achieving students," in Science and Technology Education for a Diverse World: Dilemmas, Needs and Partnerships, eds R. Janiuk, and E. Samonek-Miciuk (Lubin: Marie Curie-Sklodowska University Press), 369-384.

Moakler, M. W. Jr., and Kim, M. M. (2014). College major choice in stem: revisiting confidence and demographic factors. Career Dev. Q. 62, 128-142. doi: 10.1002/j.2161-0045.2014.00075.

Mohammadinia., T y Negahdari, S. (2017). Investigation of Factors Affecting the Cultural Capital (Case Study: Payame Noor and Islamic Azad Universities Students). American Journal of Sociological Research, 7(2): 72-76. DOI: 10.5923/j.sociology.20170702.02 
Negewo, M.A., \& Murugan, P. (2018). Cultural Capital and Students'Academic Performance: The Case of Ethiopian Higher Educational Institutions. Eastern Africa Social Science Research Review 34(1), 249-276. doi:10.1353/eas.2018.0008.

Roksa, J. y Potter, D. (2011) Parenting and academic achievement: intergenerational transmission of educational advantage. Sociology of Education, 84, 299-321.

Shapiro, T., Meschede, T., and Osoro, S. (2013). "The widening racial wealth gap," in The Assets Perspective, eds R. Cramer and T. Shanks (New York, NY: Palgrave Macmillan), 1-8.

Suhonen, T., y Karhunen, H. (2019). The intergenerational effects of parental higher education: Evidence from changes in university accessibility. Journal of Public Economics, 176, 195-217. doi:10.1016/j.jpubeco.2019.07.001

Wu, C.-L., and Bai, H. (2015). From early aspirations to actual attainment: the effects of economic status and educational expectations on university pursuit. High. Educ. 69, 331-344. doi: 10.1007/s10734-014-9778-1

Xu, J., y Hampden-Thompson, G. (2012). Cultural Reproduction, Cultural Mobility, Cultural Resources, or Trivial Effect? A Comparative Approach to Cultural Capital and Educational Performance. Comparative Education Review, 56(1), 98-124. doi:10.1086/661289

Yamamoto, Y., \& Brinton, M. C. (2010). Cultural Capital in East Asian Educational Systems. Sociology of Education, 83(1), 67-83. doi:10.1177/0038040709356567 